\title{
Semiparametric Modeling of Stochastic and Deterministic Trends and Fractional Stationarity
}

\author{
Jan Beran ${ }^{1}$, Yuanhua Feng ${ }^{1}$, Günter Franke ${ }^{2}$, Dieter Hess ${ }^{2}$, and Dirk Ocker ${ }^{3}$ \\ 1 Department of Mathematics and Statistics \\ 2 Department of Economics \\ University of Konstanz, D-78457 Konstanz, Germany \\ 3 Schweizer Verband der Raiffeisenbanken, CH-9001 St.Gallen
}

\begin{abstract}
The distinction between stationarity, difference stationarity, deterministic trends as well as between short- and long-range dependence has a major impact on statistical conclusions, such as confidence intervals for population quantities or point and interval forecasts. SEMIFAR models introduced by [6] provide a unified approach that allows for simultaneous modelling of and distinction between deterministic trends, difference stationarity and stationarity with short- and long-range dependence. In this paper, recent results on the SEMIFAR models are summarized and their potential usefulness for economic time series analysis is illustrated by analyzing several commodities, exchange rates, the volatility of stock market indices and some simulated series. Predictions combine stochastic prediction of the random part with functional extrapolation of the deterministic part.
\end{abstract}

\section{Introduction}

Many economic time series exhibit apparent local or global 'trends'. A large number of methods for dealing with trends under specific assumptions are described in the literature (see e.g. standard time series books, such as [16]; [37]. Essentially, models for trends can be classified as either (1) deterministic or (2) stochastic. A deterministic trend is described by a deterministic function $g(t)$, whereas a stochastic trend is generated by a purely stochastic nonstationary process such as random walk, (fractional) Brownian motion or an integrated ARIMA process. As a third possibility, local "spurious" trends can be generated by stationary processes with long-range dependence, such as stationary fractional ARIMA models. Statistical inference about population quantities and statistical forecasts are greatly influenced by our decision about the type of the 'trend' generating mechanism. For instance, for a stationary series, forecasts of a conditional expected value converge to the sample mean, with increasing forecasting horizon, and the width of forecast intervals is asymptotically constant. In contrast, for difference stationary series, forecasts converge to the last observation and the width of forecast intervals diverges to infinity. Forecasts for time series with a deterministic trend require reliable trend extrapolation which can usually not be trusted beyond a small forecasting horizon. On a finer scale, the rate at which forecast intervals converge to the asymptotic width (for stationary 
processes) or diverge to infinity (for difference stationary processes) depends on the fractional differencing parameter (see Sect. 4).

In practical applications, it is often very difficult to find the "right" model and, in particular, to decide whether a series is stationary, has a deterministic or stochastic trend, or whether there may be long-range correlations. In fact, often, a combination of these may be present. To resolve this problem, [6] introduced the so-called SEMIFAR (semiparametric fractional autoregressive) models. These models provide a unified data-driven semiparametric approach that allows for simultaneous modelling of and distinction between deterministic trends, stochastic trends and stationary short- and long-memory components. Within the given framework, the approach helps the data analyst to decide which components are present in the observed data. In this paper, recent results on SEMIFAR models ([6], [9], [8]) are summarized and their application to economic time series is discussed.

Briefly speaking, a SEMIFAR model is a fractional stationary or non-stationary autoregressive model with a nonparametric deterministic trend. This extends Box-Jenkins ARIMA models ( $[10]$ ), by using a fractional differencing parameter $d>-0.5$, and by including a nomparametric trend function $g$. The trend function can be estimated, for example, by kernel smoothing. The parameters may be estimated by an approximate maximum likelihood method introduced in [5]. Note in particular that, with this method the integer differencing parameter is also estimated from the data. A data-driven algorithm for estimating SEMI$F A R$ models, which is a mixture of these two approaches, was introduced in [6]. Clearly, as any statistical method, the analysis by SEMIFAR models has to be accompanied by appropriate subject-specific considerations.

The paper is organized as follows. The model is defined in Sect. 2. Estimation issues are discussed in. Sect. 3, especially nonparametric estimation of the trend and the method for estimating the parameters characterizing the stochastic component of the process. Forecasting with SEMIFAR models is described in Sect. 4. The application of SEMIFAR models to economic time series is discussed in Sect. 5. In particular, we discuss modelling and forecasting commodities and exchange rates, and modelling the volatility of stock market indices. Also, four simulated series are analyzed to illustrate the usefulness of the method for cases where the answer is known. (A broader simulation study is reported in [6]). Some final remarks are given in Sect. 6 .

\section{The Model}

\subsection{Definition}

A SEMIFAR model is a Gaussian process $Y_{i}$ with an existing smallest integer $m \in\{0,1\}$ such that

$$
\phi(B)(1-B)^{\delta}\left\{(1-B)^{m} Y_{i}-g\left(t_{i}\right)\right\}=\epsilon_{i},
$$

where $t_{i}=(i / n), \delta \in(-0.5,0.5), g$ is a smooth function on $[0,1], B$ is the backshift operator, $\phi(x)=1-\sum_{j=1}^{p} \phi x^{j}$ is a polynomial with roots outside the unit circle and $\epsilon_{i}(i=\ldots,-1,0,1,2, \ldots)$ are iid zero mean normal with var $\left(\epsilon_{i}\right)=$ $\sigma_{\epsilon}^{2}$. Here, the fractional difference $(1-B)^{\delta}([25],[30])$ is defined by

$$
(1-B)^{\delta}=\sum_{k=0}^{\infty} b_{k}(\delta) B^{k}
$$

with

$$
b_{k}(\delta)=(-1)^{k} \frac{\Gamma(\delta+1)}{\Gamma(k+1) \Gamma(\delta-k+1)}
$$

\subsection{Intuitive Explanation of the Definition}

The motivation for this definition can be summarized as follows: We wish to have a model that may be decomposed into an arbitrary deterministic (possibly zero) trend and a random component that may be stationary or difference stationary. Moreover, short-range and long-range dependence as well as antipersistence should be included. Here, long-range dependence is defined as follows (see, e.g. [35]; [14]; [27]; [31]; [4] and references therein): A stationary process $Y_{i}$ with autocovariances $\gamma(k)=\operatorname{cov}\left(Y_{i}, Y_{t+k}\right)$ is said to have long-range dependence, if the spectral density $f(\lambda)=(2 \pi)^{-1} \sum_{k=-\infty}^{\infty} \exp (i k \lambda) \gamma(k)$ has a pole at the origin of the form

$$
f(\lambda) \sim c_{f}|\lambda|^{-\alpha}(|\lambda| \rightarrow 0)
$$

for a constant $c_{f}>0$ and $\alpha \in(0,1)$, where $\sim$ " means that the ratio of the left and right hand sides converges to one. In particular, this implies that, as $k \rightarrow \infty$, the autocovariances $\gamma(k)$ are proportional to $k^{\alpha-1}$ and hence their sum is infinite. On the other hand, a stationary process is called antipersistent, if (4) holds with $\alpha \in(-1,0)$. This implies that the sum of all autocovariances is zero, i.e. $\sum_{k=-\infty}^{\infty} \gamma(k)=0$. Note that for usual short-memory processes, such as stationary ARMA processes, (4) holds with $\alpha=0$, and the autocovariances sum up to a nonzero finite value.

The reason for including long-memory and anti-persistence is that for traditional ARIMA models an extreme choice has to be made between taking no or the first difference. The result of this dichotomy is that for many data sets, taking no difference is not enough (i.e. the series seems nonstationary), but taking the first difference leads to overdifferencing. The latter often results in a large negative lag-one correlation for the differenced data. To avoid this and to model slowly decaying correlations, [30]) and [25] introduced fractional ARIMA processes. However, there, the differencing parameter $d$ is restricted to the stationarity region $(-1 / 2,1 / 2)$. In a direct extension, [5] defines an arbitrary differencing parameter $d>-1 / 2$ such that $(1-B)^{m} Y_{t}$ is a stationary fractional $\operatorname{ARIMA}(p, \delta, q)$ process, $m=[d+1 / 2]$ is the integer part of $d+1 / 2$ and $\delta=d-m$. $T$ This corresponds to (1) with a constant function $g \equiv \mu$. Since the integer differencing parameter $m$ assumes integer values only and the fractional differencing parameter $\delta$ is in $(-1 / 2,1 / 2)$, both differencing parameters can be recovered uniquely from the 'overall differencing parameter' $d=m+\delta$. If $d>1 / 2$, then 
we have a nonstationary fractional ARIMA process. It should be noted, in particular, that this parameterisation allows for maximum likelihood estimation of $d$. Thus not only $\delta$, but also $m$ can be estimated from the data and confidence intervals can be given for both differencing parameters (see [5])

Finally, SEMIFAR models extend the definition of fractional ARIMA models with arbitrary $d$ by including an arbitrary deterministic trend function $g$. (For simplicity only cases with $q=0$ (i.e. no moving average terms) are considered. An extension to $q>0$, which may be called 'SEMIFARIMA models', is obvious.) The definition of SEMIFAR models includes all the desired cases mentioned above. In particular, setting $\delta=0$ and $g(t)=\mu$, we obtain classical Box-Jenkins ARIMA models. For $g=0$, and $m=0$ we have stationary fractional ARIMA models as defined in [30] and [25].

More specifically, for SEMIFAR models, $Z_{i}=\left\{(1-B)^{m} Y_{i}-g\left(t_{i}\right)\right\}$ is a stationary fractional autoregressive process. Thus, the spectral density of $Z_{i}$ is proportional to $|\lambda|^{-2 \delta}$ at the origin so that the process $\left\{(1-B)^{m} Y_{i}-g\left(t_{i}\right)\right\}$ has long-memory if $\delta>0$, antipersistence if $\delta<0$ and short memory if $\delta=$ 0 . (1) generalizes stationary fractional AR-processes to the nonstationary case, including difference stationarity and deterministic trend. The following special cases are thus included in (1):

(a) $Y_{t}=$ no deterministic trend + stationary process with short- or long-range dependence, or antipersistence;

(b) $Y_{t}=$ deterministic trend + stationary process with short- or long-range dependence, or antipersistence;

(c) $Y_{t}=$ no deterministic trend + difference-stationary process, whose first difference has short- or long-range dependence, or antipersistence;

(d) $Y_{t}=$ deterministic trend + difference-stationary process, whose first difference has short- or long-range dependence, or antipersistence.

Simulated time series for these special cases are shown in Fig. 1, where Figs. 1a to 1d correspond to case (a) trough (d), respectively. A full description of the models used in figures la to d is given in Sect. 5.2

\subsection{Some Economic Motivation}

Since the estimation of the SEMIFAR-model is purely data-driven, there exists a danger that the estimated model is inconsistent with economic reasoning. If this happens to be true, then the estimated model and the economic reasoning are called into question. In the following, we will briefly discuss some economic models which can explain short and long-term dependence in time series of prices of commodities and financial securities.

The implications of pricing models necessarily depend on the assumptions made. Many models assume perfect markets and perfectly rational economic agents. A basic requirement for any viable model is that it precludes arbitrage A market can be arbitrage-free only if all prices for state-contingent claims are positive and finite. Let $S_{t}$ be the price of some security at date $t$. For example, consider a stock whose price may be considered the risk-adjusted present
First simulated series

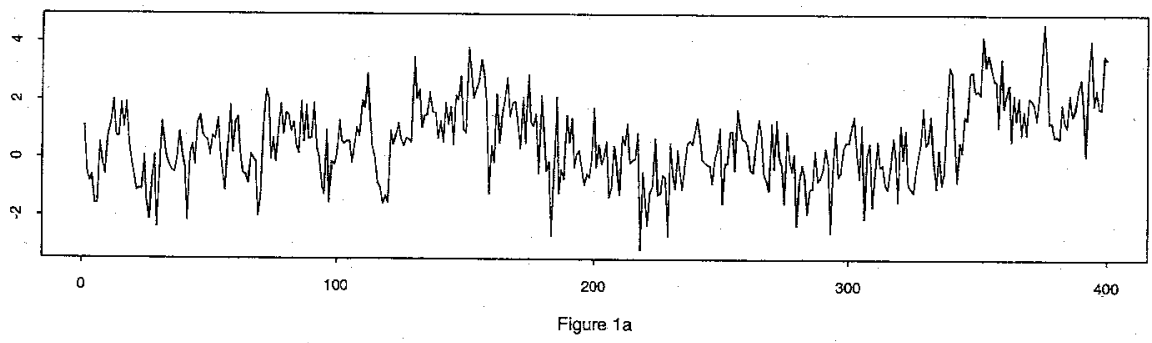

Second simulated series

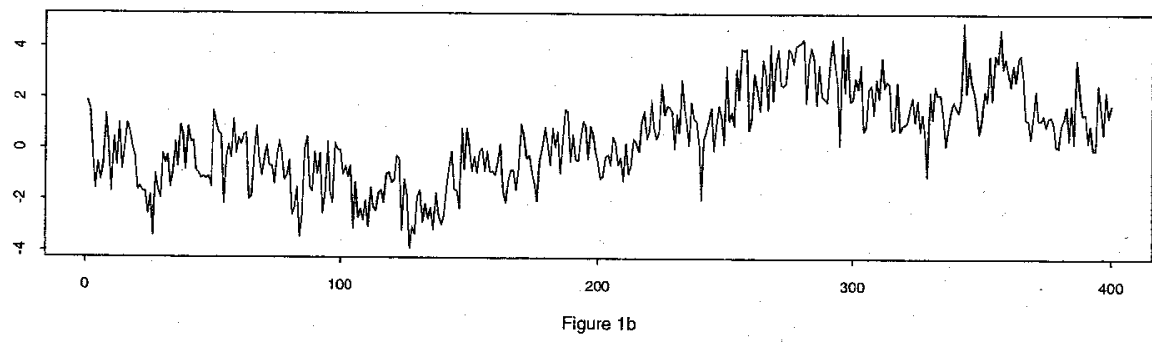

Third simulated series

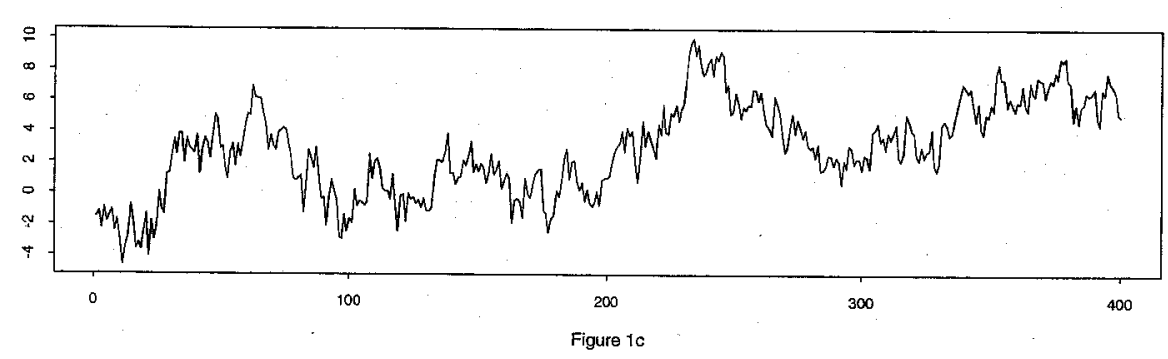

Fourth simulated series

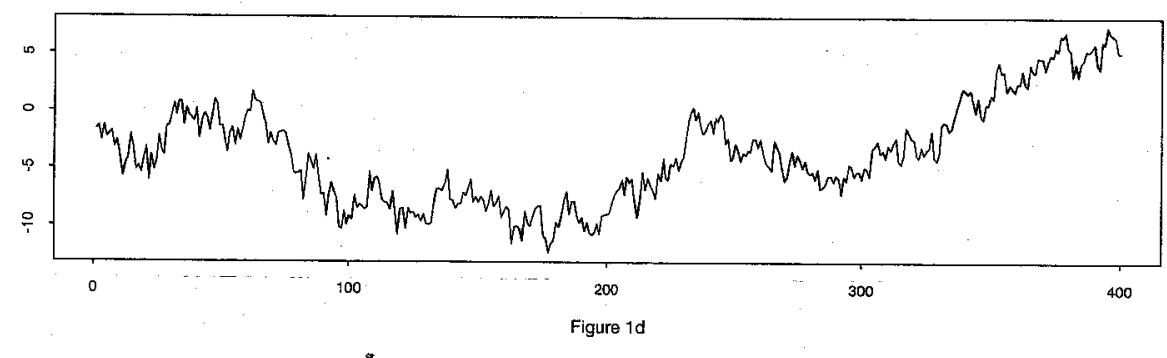

Fig. 1. Simulated series 
value of future dividends. Suppose that there exist exogenous shocks at date 0 which increase (reduce) all future dividends $d_{t},(t=1,2, \ldots)$ by finite amounts. Then, given sufficiently low discount rates, the stock price would change by an infinite amount. Hence, the market would not satisfy the no-arbitrage requirement ([34]). As Mandelbrot points out, fractional Brownian motions, which are typical stochastic models with long-range dependence or antipersistence (in the increment process), do not rule out these cases.

Another violation of the no-arbitrage requirement is obtained if the short run-autocorrelation of price changes is very high or very low. Then an observed price change would permit an almost riskless forecast of the price change over the next time period which then could be arbitraged against a risk-free asset. [39] also proves the existence of arbitrage opportunities in fractional Brownian motions, but he also shows that a slight modification of the model suffices to rule out these opportunities. Note, in particular, that according to Mandelbrot's definition, arbitrage exists for all long-memory processes whereas this is not the case according to Rogers' definition. Thus, the answer to the question whether arbitrage is possible depends on which definition of arbitrage is used.

Even if arbitrage opportunities do not exist, the economist wonders how any short or long range dependencies in price series might be explained. [40] has shown that prices must follow a random walk in a risk neutral world with a non-random risk-free interest rate. For simplicity, consider an asset with an exogenously given random price $S_{T}$ at date $T$. Then in a risk neutral world with homogeneous expectations of economic agents, the forward price $S_{t}^{f}$ of the asset at date $t$ equals $E_{t}\left[S_{T}\right]$, i.e. the conditional expectation of $S_{T}$. The forward price eliminates, by definition, the discounting effects of the risk-free interest rates. Since the conditional expectation of $S_{T}$ follows a random walk without drift, any dependencies in forward price changes are ruled out.

In a risk-averse world with a frictionless complete market, there exists unique forward pricing kernel $\phi_{t, T}\left(S_{T}\right)$ at date $t(t<T)$, by which the forward price $S_{t}^{f}$ can be derived. We have $E\left[\phi_{t, T}\left(S_{T}\right)\right]=1$ and $S_{T}^{f}=E_{t}\left[\phi_{t, T}\left(S_{T}\right) S_{T}\right]$ Then there still exist cases in which dependencies in forward price changes do not exist. Suppose, for example, that $E_{t}\left(S_{T}\right)$ follows a standard geometric Brownian motion without drift. Then $\ln \left(S_{t+1}^{f} / S_{t}^{f}\right)$ follows a standard geometric Brownian motion with drift if and only if the forward pricing kernel has constant elasticity $\eta_{t, T}$, i.e. if $d \ln \phi_{t, T} / d \ln S_{T}=\eta_{t, T}, \forall t$ ([20]). Now suppose that the elasticity depends on $S_{T}$, holding the current forward price $S_{0}^{f}$ constant. Suppose that $d \eta_{t, T} / d S_{T}<0$ which may be thought of as "declining relative risk aversion of the market". Then the variance of the forward price $S_{t}^{f}$ increases and the $\log$ returns $\ln \left(S_{t+1}^{f} / S_{t}^{f}\right)$ are negatively autocorrelated. In the case of increasing elasticity of the pricing kernel, the variance of the forward price declines relative to the constant elasticity case, but autocorrelation of log returns still is negative. The intuition behind this result is straightforward. Whenever the forward price $S_{t}^{f}$ is higher or lower, relative to the constant elasticity case which implies zero autocorrelation, then given $S_{0}^{f}$ and the distribution of $S_{T}$, a lower return in one period must be compensated by a higher return in the residual period. This implies short and long-range negative autocorrelation. Hence, in this framework, antipersistence may exist whereas it is difficult to argue in favor of positive autocorrelation.

Of course, real markets are not perfect. Introducing asymmetric information broadens the spectrum of return processes. Insiders, for example, attempt to exploit their information privilege by strategic trading which leads to a gradual price adjustment and, thus, short range positive autocorrelation of returns. The same autocorrelation is to be expected in the case of positive feedback trading. Then agents observe a price increase and place additional buy orders since they expect a further price increase. Finally, if large investors buy or sell consecutively small portions of a rather illiquid security, this induces positive autocorrelation.

[34] suggests that economic agents have a finite foresight horizon. This may imply various dependencies in returns. If agents, for example, ignore effects of a shock on a corporation's profits beyond some horizon, then these effects will gradually be taken into consideration and generate long-range dependent price changes. Alternatively, if agents naively extrapolate growth rates of profits over very long time horizons, this extrapolation error will gradually be corrected with corresponding gradual price changes. Such behavior might explain the well documented winner-loser effect which states that stocks with high returns over the last years tend to generate low returns over the next years and vice versa. Also cyclical macroeconomic factors tend to generate cyclical stock price behavior given either a short foresight horizon or naive extrapolative behavior. Otherwise it would be useless to distinguish between cyclical and noncyclical stocks.

Similar considerations apply to commodities for which short and long rangecontracts are traded. The economic analysis of commodity prices becomes more complicated since durability of commodities and side effects of storing commodities summarized in convenience yields come into play.

Finally, long memory in aggregated indices may be a result of aggregation. As was shown, for instance, in [23], adding up a large number of time series can lead (asymptotically) to a series with long-range dependence, even if the individual series do not exhibit any long memory.

Yet, adding these real world aspects to purely theoretical models should not be understood as providing unlimited freedom to all kinds of short and longrange dependencies in time series of prices. The ultimate purpose of research is to find out price processes that are observable with sufficient reliability and are grounded on solid economic reasoning. SEMIFAR models provide a rather general class of models to do the empirical job. The economists should use the empirical insights for developing sensible economic models.

\section{Estimation of SEMIFAR Models - A Review}

Estimation of SEMIFAR models includes (1) nonparametric estimation of the trend component and (2) estimation of the parameters characterizing the stochastic component. This section summarizes theoretical results on the proposed kernel estimator of the trend function and approximate maximum likelihood 
estimator of the parameters without proofs. See [6] and [8] for details. A datadriven algorithm for estimating the whole model is also briefly described.

\subsection{Kernel Estimation of the Trend Function}

The problem of estimating $g$ from data given by

$$
Y_{i}=g\left(t_{i}\right)+X_{i}
$$

has been considered by various authors for the case where the error process $X_{t}$ is stationary with (i) short-range dependence, i.e. (4) holds with $\alpha=0$ (see e.g. [13], [2], [26] and [29]) or (ii) long-range dependence, i.e. $0<\alpha<1$ (see e.g. [26]; [15] and [38]). For SEMIFAR models defined by (1), the cases (i) and (ii) are obtained by setting $m=0$ and $\delta=\alpha / 2=0$ (case (i)), or $m=0$ and ' $\delta \in(0,1 / 2)$ (case (ii)) respectively. For $m=1$, the same is true for the first difference $Y_{i}-Y_{i-1}$. (Note, however, that for SEMIFAR models, $m \in\{0,1\}$ is an unknown parameter!) In addition to cases (i) and (ii), definition 1 also includes the antipersistent case, i.e. $\delta<0$ so that the spectral density $f$ of $Y_{i}$ (or $Y_{i}-Y_{i-1}$ respectively) converges to zero at the origin. The theorem below extends previous results on kernel estimation to the anti-persistent case, and gives formulas for the mean squared error and the optimal bandwidth that are valid for the whole range $\delta \in(-0.5,0.5)$.

For estimating $g$ by kernel smoothing, symmetric polynomial kernels of the form $K(x)=\left\{\sum_{l=0}^{r} \alpha_{l} x^{2 l}\right\} \mathbb{I}_{\{|x| \leq 1\}}$ (see e.g. [21]) will be used. If (5) holds, then, for a given bandwidth $b>0$ and $t \in[0,1]$, the kernel estimate of $g$ is defined by

$$
\hat{g}(t)=K_{b} \diamond y(n)=\frac{1}{n b} \sum_{i=1}^{n} K\left(\frac{t-t_{i}}{b}\right) Y_{i}
$$

where $y(n)=\left(Y_{1}, \ldots, Y_{n}\right)$. Let $n_{0}=[n t], n_{1}=[n b]$ and $0<\Delta<0.5$, the following notations will be used:

$$
\begin{gathered}
V_{n}(\theta, b)=(n b)^{-1-2 \delta} \sum_{i, j=n_{0}-n_{1}}^{n_{0}+n_{1}} K\left(\frac{t-t_{i}}{b}\right) K\left(\frac{t-t_{j}}{b}\right) \gamma(i-j) \\
I\left(g^{\prime \prime}\right)=\int_{\Delta}^{1-\Delta}\left[g^{\prime \prime}(t)\right]^{2} d t
\end{gathered}
$$

and

$$
I(K)=\int_{-1}^{1} x^{2} K(x) d x .
$$

The following result is obtained under the assumption that (5) holds and that $g$ is at least twice continuously differentiable (see [6] for the proof).

Theorem 1. Let $b_{n}>0$ be a sequence of bandwidths such that $b_{n} \rightarrow 0$ and $n b_{n} \rightarrow \infty$. Then, under the stated assumptions and $\delta$ in (1) in the interval $(-0.5,0.5)$, we have (i) Bias:

$$
E[\hat{g}(t)-g(t)]=b_{n}^{2} \frac{g^{\prime \prime}(t) I(K)}{2}+o\left(b_{n}^{2}\right)
$$

(ii)

uniformly in $\Delta<t<1-\Delta$

$$
\lim _{n \rightarrow \infty} V_{n}\left(\theta, b_{n}\right)=V(\theta)
$$

where $0<V(\theta)<\infty$ is a constant;

(iii) Variance:

$$
\left(n b_{n}\right)^{1-2 \delta} \operatorname{var}(\hat{g}(t))=V(\theta)+o(1)
$$

uniformly in $\Delta<t<1-\Delta$;

(iv) IMSE: The integrated mean squared error in $[\Delta, 1-\Delta]$ is given by

$$
\begin{gathered}
\int_{\Delta}^{1-\Delta} E\left\{[\hat{g}(t)-g(t)]^{2}\right\} d t=I M S E_{\text {asympt }}\left(n, b_{n}\right)+o\left(\max \left(b_{n}^{4},\left(n b_{n}\right)^{2 \delta-1}\right)\right) \\
=b_{n}^{4} \frac{I\left(g^{\prime \prime}\right) I^{2}(K)}{4}+\left(n b_{n}\right)^{2 \delta-1} V(\theta)+o\left(\max \left(b_{n}^{4},\left(n b_{n}\right)^{2 \delta-1}\right)\right)
\end{gathered}
$$

(v) Optimal bandwidth: The bandwidth that minimizes the asymptotic IMSE is given by

where

$$
b_{o p t}=C_{o p t} n^{(2 \delta-1) /(5-2 \delta)}
$$

$$
C_{o p t}=C_{o p t}(\theta)=\left(\frac{(1-2 \delta) V(\theta)}{I\left(g^{\prime \prime}\right) I^{2}(K)}\right)^{1 /(5-2 \delta)}
$$

Similar results can be obtained for kernel estimates of derivatives of $g$. For instance, the second derivative can be estimated by $\hat{g}^{\prime \prime}(t)=n^{-1} b^{-3} \sum K\left(\left(t_{j}-\right.\right.$ t)/b) $Y_{j}$ where $K$ is a symmetric polynomial kernel such that $\int K(x) d x=0$ and $\int K(x) x^{2} d x=2$. By analogous arguments, the optimal bandwidth is then of the order $O\left(n^{(2 \delta-1) /(9-2 \delta)}\right)$

Simple explicit formulas for $V(\theta)$ can be given for $\delta=0$ and $\delta>0$ as follows (see e.g. [26]):

$$
\begin{gathered}
V(\theta)=2 \pi c_{f} \int_{-1}^{1} K^{2}(x) d x,(\delta=0) \\
V(\theta)=2 c_{f} I(1-2 \delta) \sin \pi \delta \int_{-1}^{1} \int_{-1}^{1} K(x) K(y)|x-y|^{2 \delta-1} d x d y,(\delta>0) .
\end{gathered}
$$

In order to obtain a similar formula for $\delta<0$, at a point $x$ let $K(y)=$ $\sum_{l=0}^{r} \beta_{l}(x)(x-y)^{l}=: K_{0}(x)+K_{1}(x-y)$, where $K_{0}(x)=\beta_{0}(x), K_{1}(x-y)=$ $\sum_{l=1}^{r} \beta_{l}(x)(x-y)^{l}$. Then we have (see [8])

$$
\begin{aligned}
V(\theta)= & 2 c_{f} \Gamma(1-2 \delta) \sin (\pi \delta) \int_{-1}^{1} K(x) \times \\
& \left\{\int_{-1}^{1} K_{1}(x-y)|x-y|^{2 \delta-1} d y-\int_{|y|>1} K_{0}(x)|x-y|^{2 \delta-1} d y\right\} d x
\end{aligned}
$$


for $\delta<0$. For the box-kernel (i.e. $r=0$ ), formulas $(16),(17)$ and (18) give the same result

$$
V=\frac{2^{2 \delta} c_{f} \Gamma(1-2 \delta) \sin (\pi \delta)}{\delta(2 \delta+1)}
$$

with $V(0)=\lim _{\delta \rightarrow 0} V(\delta)=\pi c_{f}$ (see corollary 1 in $[6]$ ).

\subsection{Maximum Likelihood Estimation}

The maximum likelihood estimation proposed by [5] for a constant function $g=$ $\mu$ can be carried over directly to SEMIFAR models with time-deterministic trend functions (see [6]). In particular, from the 'overall differencing parameter' $d=$ $m+\delta$ both, the discrete differencing parameter $m$ and the fractional differencing parameter $\delta$ can be recovered uniquely, since $m$ can take on integer values only and $\delta$ is in $(-1 / 2,1 / 2)$. Moreover, this parameterization allows for maximum likelihood estimation of $d$ (and thus of $\delta$ and $m$ ) along with the autoregressive parameters and the trend function. Moreover, inference about the autoregressive parameters takes into account that $m$ and $\delta$ were not known a priori.

Let $\theta^{\circ}=\left(\sigma_{\epsilon, o}^{2}, d^{o}, \phi_{1}^{o}, \ldots, \phi_{p}^{o}\right)^{T}=\left(\sigma_{\epsilon, o}^{2}, \eta^{o}\right)^{T}$ be the true unknown parameter vector in (1) where $d^{o}=m^{o}+\delta^{o},-1 / 2<\delta^{\circ}<1 / 2$ and $m^{\circ} \in\{0,1\}$. Then

$$
\begin{aligned}
\phi(B)(1-B)^{\delta^{\circ}}\left\{(1-B)^{m^{o}} Y_{i}-g\left(t_{i}\right)\right\} & =\sum_{j=0}^{\infty} a_{j}\left(\eta^{o}\right) B^{j}\left[c_{j}\left(\eta^{o}\right) Y_{i}-g\left(t_{i}\right)\right] \\
& =\sum_{j=0}^{\infty} a_{j}\left(\eta^{o}\right)\left[c_{j}\left(\eta^{o}\right) Y_{i-j}-g\left(t_{i-j}\right)\right]
\end{aligned}
$$

where the coefficients $a_{j}$ and $a_{j} c_{j}$ are obtained by matching the powers in $B$. Hence, $Y_{i}$ admits an infinite autoregressive representation

$$
\sum_{j=0}^{\infty} a_{j}\left(\eta^{o}\right)\left[c_{j}\left(\eta^{o}\right) Y_{i-j}-g\left(t_{i-j}\right)\right]=\epsilon_{i}
$$

Let $b_{n}(n \in N)$ be a sequence of positive bandwidths such that $b_{n} \rightarrow 0$ and $n b_{n} \rightarrow \infty$ and define $\hat{g}\left(t_{i}\right)=\hat{g}\left(t_{i} ; m\right)$ by

$$
\hat{g}\left(t_{i} ; 0\right)=K_{b_{n}} \diamond y(n)
$$

and

$$
\hat{g}\left(t_{i} ; 1\right)=K_{b_{n}} \diamond D y(n),
$$

with $D y(n)=\left(Y_{2}-Y_{1}, Y_{3}-Y_{2}, \ldots, Y_{n}-Y_{n-1}\right)$. Consider now $\epsilon_{i}$ as a function of $\eta$. For a chosen value of $\theta=\left(\sigma_{\epsilon}^{2}, m+\delta, \phi_{1}, \ldots, \phi_{p}\right)^{T}=\left(\sigma_{\epsilon}^{2}, \eta\right)^{T}$, denote by

$$
e_{i}(\eta)=\sum_{j=0}^{i-m-2} a_{j}(\eta)\left[c_{j}(\eta) Y_{i-j}-\hat{g}\left(t_{i-j} ; m\right)\right]
$$

the (approximate) residuals and by $r_{i}(\theta)=e_{i}(\eta) / \sqrt{\theta_{1}}$ the standardized residuals. Assuming that $\left\{\epsilon_{i}\left(\eta^{o}\right)\right\}$ are independent zero mean normal with variance $\sigma_{\epsilon, o}^{2}$, an approximate maximum likelihood estimator of $\theta^{\circ}$ is obtained by maximizing the approximate log-likelihood

$$
l\left(Y_{1}, \ldots, Y_{n} ; \theta\right)=-\frac{n}{2} \log 2 \pi-\frac{n}{2} \log \sigma_{\epsilon}^{2}-\frac{1}{2} n^{-1} \sum_{i=m+2}^{n} r_{i}^{2}
$$

with respect to $\theta$ and hence by solving the equations

$$
i\left(Y_{1}, \ldots, Y_{n} ; \theta\right)=0
$$

where $l$ is the vector of partial derivatives with respect to $\theta_{j}(j=1, \ldots, p+2)$. More explicitly, $\hat{\eta}$ is obtained by minimizing

$$
S_{n}(\eta)=\frac{1}{n} \sum_{i=m+2}^{n} e_{i}^{2}(\eta)
$$

with respect to $\eta$ and setting

$$
\hat{\sigma}_{\epsilon}^{2}=\frac{1}{n} \sum_{i=m+2}^{n} e_{i}^{2}(\hat{\eta}) .
$$

The result in [5] can be extended to SEMIFAR models [6]:

Theorem 2. Let $\hat{\theta}$ be the solution of (26) and (27), and define $\theta_{*}^{o}=\left(\sigma_{\epsilon, o}^{2}, \eta_{*}^{o}\right)^{T}$ $=\left(\sigma_{\epsilon, o}^{2}, \delta^{o}, \eta_{2}^{o}, \ldots, \eta_{p+1}^{o}\right)^{T}$. This means that, $\theta_{2}^{o}=d=m^{o}+\delta^{o}$ is replaced by $\theta_{2, *}^{o}=\delta^{o}$. Then, as $n \rightarrow \infty$,

(i) $\hat{\theta}$ converges in probability to the true value $\theta^{\circ}$;

(ii) $n^{\frac{1}{2}}\left(\hat{\theta}-\theta^{\circ}\right)$ converges in distribution to a normal random vector with mean zero and covariance matrix

$$
\Sigma=2 D^{-1}
$$

where

$$
D_{i j}=\left.(2 \pi)^{-1}\left[\int_{-\pi}^{\pi} \frac{\partial}{\partial \theta_{i}} \log f(x) \frac{\partial}{\partial \theta_{j}} \log f(x) d x\right]\right|_{\theta=\theta_{*}^{o} .}
$$

It should be noted that in theorem 2 , both, the fractional differencing parameter $\delta$ and the integer differencing parameter $m$ are estimated from the data. The asymptotic covariance matrix does not depend on $m$. Theorem 2 can be generalized to the case where the innovations $\epsilon_{i}$ are not normal, and satisfy suitable moment conditions.

Theorem 2 is derived under the assumption that the order $p=p_{o}$ of the autoregressive polynomial in (1) is known. In practice $p_{o}$ needs to be estimated by applying a suitable model choice criterion. It can be shown, however, that consistency properties of model choice criteria, such as the BIC ([41]; [1]) and the HIC ([28]), are analogous to the case of stationary short-memory autoregressive processes $([6])$ : 
Theorem 3. Under the assumptions of theorem 2, let $p_{0}$ be the true order of the polynomial $\phi$ in (1) and define

$$
\hat{p}=\arg \min \left\{A I C_{\alpha}(p) ; p=0,1, \ldots, L\right\}
$$

where $L$ is a fixed integer, $A I C_{\alpha}(p)=n \log \hat{\sigma}_{\epsilon}^{2}(p)+\alpha \cdot p$ and $\hat{\sigma}_{\epsilon}^{2}(p)$ is the maximum likelihood estimate of the innovation variance $\sigma_{\epsilon, o}^{2}$ using a SEMIFAR model with autoregressive order $p$. Moreover, define $\hat{\theta}$ by (26) and (27) with $p$ set equal to $\hat{p}$. Suppose furthermore that $\alpha$ is at least of the order $O(2 c \log \log n)$ for some $c>1$. Then the results of theorem 2 hold.

Combining Theorems 1 through 3, It is straightforward to obtain from confidence intervals for the unknown parameter vector $\theta$ and the unknown trend function $g$, as well as for testing hypotheses about $\theta$ and $g$. Note, in particular, that the integer differencing parameter $m$ is also estimated by maximum likelihood ( $\hat{m}$ is equal to the integer part of $\hat{d}+1 / 2$ ).

\subsection{Estimation of the Whole Model}

For estimating the whole model one needs a semiparametric data-driven algorithm combining the two estimation methods described above. An algorithm for the case where $g$ is assumed to be equal to a constant $\mu$ is given in [5]. A data-driven algorithm for estimating the SEMIFAR model with a general trend function $g$ was proposed by Beran in 1997 in the original, unpublished paper on the SEMIFAR model. What follows is a brief description of this algorithm.

The algorithm makes use of the fact that $d$ is the only additional parameter, in addition to the autoregressive parameters, so that a systematic search with respect to $d$ can be made. This algorithm can be adapted to the case where $g$ is an unknown function, by replacing $\hat{\mu}$ by a kernel estimate of $g$. The optimal bandwidth can be estimated by an iterative plug-in method similar to the one in [29] and [38]. These authors consider the case of stationary errors, i.e. $m$ is known to be equal to zero. The algorithm in [38] is as follows:

1. an initial bandwidth is defined;

2. a preliminary estimate of $g$ is computed and subtracted from the observations;

3. the relevant parameters of the error process are estimated from the residuals; 4. the bandwidth is updated.

Steps 2 to 4 are repeated until the change in the bandwidth is below a predefined threshold. This algorithm has been extended to fitting SEMIFAR models ([6]). A detailed study on the consistency, rates of convergence and comparison of different iterative algorithms for SEMIFAR fitting will be given in a forthcoming paper.

\section{SEMIFAR Forecasting}

This section describes out-of-sample predictions of SEMIFAR processes. Let $Y_{1}, \ldots, Y_{n}$ be observations generated by a SEMIFAR model of order $p$ with parameter vector $\theta=\left(\sigma_{\epsilon}^{2}, d, \phi_{1}, \ldots, \phi_{p}\right)^{T}$ (where $d=m+\delta$ ). The aim is to predict a future observation $Y_{n+k}$ for some $k \in\{1,2,3, \ldots\}$. Denote by $X_{i}$ a zero mean fractional AR process of order $p$ with parameter vector $\theta_{*}=\left(\sigma_{\epsilon}^{2}, \delta, \eta_{2}, \ldots, \eta_{p+1}\right)^{T}$, and define $t_{n+k}=(n+k) / n=t_{n}+k / n$. Then

$$
Y_{n+k}=\mu\left(t_{n+k}\right)+U_{n+k}
$$

with

$$
\mu\left(t_{n+k}\right)=g\left(t_{n+k}\right), \quad U_{n+k}=X_{n+k}
$$

if $m=0$, and

$$
\mu\left(t_{n+k}\right)=Y_{n}+\sum_{j=1}^{k} g\left(t_{n+j}\right), \quad U_{n+k}=\sum_{j=1}^{k} X_{n+j}
$$

if $m=1$. Thus, to predict $Y_{n+k}$ from $Y_{1}, \ldots, Y_{n}$, two problems need to be solved:

1. extrapolation of the function $\mu(t)$ to $t=t_{n+k}$;

2. prediction of the stochastic component $U_{n+k}$.

\subsection{Extrapolation of the Trend Function}

Since for SEMIFAR models only general regularity conditions on $g$ are imposed, the deterministic trend $g(t)$ may behave in an arbitrary way in the future. This is in contrast to parametric trend models. However, we may obtain the predictions of $\hat{g}\left(t_{n+j}\right)$ for $j \in\{1,2, \ldots, k\}$, for instance by a local constant or a local linear extension of $\hat{g}\left(t_{n}\right) . \hat{\mu}\left(t_{n+k}\right)$ is obtained by inserting $\hat{g}\left(t_{n+k}\right)$ in $(32)$ or $\hat{g}\left(t_{n+j}\right)$ for $j \in\{1,2, \ldots, k\}$ in (33) (see [9]).

\subsection{Prediction of the Stochastic Component}

Note that $X_{i}=U_{i}=Y_{i}-g\left(t_{i}\right)$ for $m=0$, and $X_{i}=U_{i}-U_{i-1}=Y_{i}$ $Y_{i-1}-g\left(t_{i}\right)$ for $m=1$. Let $\gamma(k)=\operatorname{cov}\left(X_{i}, X_{i+k}\right)$ denote the autocovariances of $X_{i}$. Using the mean square criterion, the best linear predictor of $U_{n+k}$ based on $Y_{1}, \ldots, Y_{n}$ is defined by $\hat{U}_{n+k}=\beta_{o p t}^{T} X(n)$ where $X(n)=\left(X_{1}, \ldots, X_{n}\right)^{T}$ and the vector $\beta_{o p t}=\left(\beta_{1}, \ldots, \beta_{n}\right)^{T}$ minimizes the mean squared prediction error $M S E=E\left[\left(U_{n+k}-\hat{U}_{n+k}\right)^{2}\right]$. The values of $\beta_{o p t}$ and the corresponding optimal mean squared prediction error $M S E_{\text {opt }}$ are given by ([9])

Theorem 4. For all integers $r, s>0$, define

$$
\gamma_{r}^{(s)}=[\gamma(r+s-1), \gamma(r+s-2), \ldots, \gamma(r)]^{T}
$$




$$
\tilde{\gamma}_{k}^{(n)}=\sum_{j=1}^{k} \gamma_{j}^{(n-1)}
$$

and denote by $\Sigma_{n}=[\gamma(i-j)]_{i, j=1, \ldots, n}$ the covariance matrix of $X(n)$. Then, the following holds.

i) If $m=0$,

$$
\begin{gathered}
\beta_{o p t}=\Sigma_{n}^{-1} \gamma_{k}^{(n)} \\
M S E_{o p t}=\gamma(0)-\left[\gamma_{k}^{(n)}\right]^{T} \Sigma_{n}^{-1}\left[\gamma_{k}^{(n)}\right]
\end{gathered}
$$

ii) If $m=1$,

$$
\begin{gathered}
\beta_{o p t}=\Sigma_{n}^{-1} \tilde{\gamma}_{k}^{(n)} \\
M S E_{o p t}=\sum_{s=-(k-1)}^{k-1}(k-|s|) \gamma(s)-\left[\tilde{\gamma}_{k}^{(n)}\right]^{T} \Sigma_{n}^{-1}\left[\tilde{\gamma}_{k}^{(n)}\right] .
\end{gathered}
$$

Note in particular that, as $k \rightarrow \infty$, the MSE tends to a finite constant in the case of a stationary stochastic component $(m=0)$, whereas it diverges to infinity in the case of a nonstationary stochastic component $(m=1)$. More specifically we have ([9])

Corollary 3. Define $c_{f}=\lim _{\lambda \rightarrow 0}|\lambda|^{2 \delta} f(\lambda)$ where $f$ is the spectral density of $X_{i}$, and let

$$
\nu(\delta)=\frac{2 \Gamma(1-2 \delta) \sin \pi \delta}{\delta(2 \delta+1)}
$$

for $0<|\delta|<0.5$ and $\nu(0)=\lim _{\delta \rightarrow 0} \nu(\delta)=2 \pi$. Then, as $k \rightarrow \infty$, the following holds:

$$
\begin{aligned}
& \text { i) If } m=0, \\
& \text { ii) If } m=1,
\end{aligned} \quad M S E_{\text {opt }} \rightarrow \gamma(0)=\operatorname{var}\left(X_{i}\right) \text {; }
$$

$$
M S E_{\text {opt }} \sim c_{f} \nu(\delta) k^{1+2 \delta} .
$$

Note in particular that, for $m=1$ and $\delta<0$, the $M S E_{\text {opt }}$ diverges to infinity at a slower rate than in the case of a random walk (with $\delta=0$ ). Similarly, for $m=1$ and $\delta>0$, the $M S E_{\text {opt }}$ diverges faster to infinity.

\subsection{Prediction Intervals}

Results in theorem 4 and corollary 1 can be used to obtain prediction intervals for $Y_{n+k}$ with $k>1$.. For known values of $g$ and $\theta$ a $100(1-\alpha)$.percent prediction interval for $Y_{n+k}$, is given by

$$
\hat{Y}_{n+k} \pm z_{\alpha / 2} \sqrt{M S E_{o p t}}
$$

where $\hat{Y}_{n+k}=\mu\left(t_{n+k}\right)+\beta_{o p t}^{T} X(n)$ and the values of $\beta_{o p t}$ and $M S E_{\text {opt }}$ are obtained from theorem 1 . If $g$ and $\theta$ are estimated, the quantities in (43) are replaced by the corresponding estimated quantities.

\section{$5 \quad$ Examples}

In this section we provide some insight into the empirical validity of the SEMIFAR models by analyzing some price series and some index volatility series. Moreover, some simulation exercises demonstrate the model's capacity to find out the true properties of a time series.

\subsection{Commodities and Exchange Rates}

The data (Figs. 2a to d) include daily spot prices for copper (between January 2, 1997, and September 2, 1998, $n=421$ ), a monthly price series for cocoa beans (between January 1971 and September 1996, $n=310$ ), and two daily nominal exchange rates (between September 17, 1997, and August 4, 1998, $n=221$ ). The currencies are the Swiss Franc (chf) and the European Currency. Unit (xeu). The data are expressed in US dollars per unit of the corresponding series. The log-transformation (natural logarithm) was applied to each series.
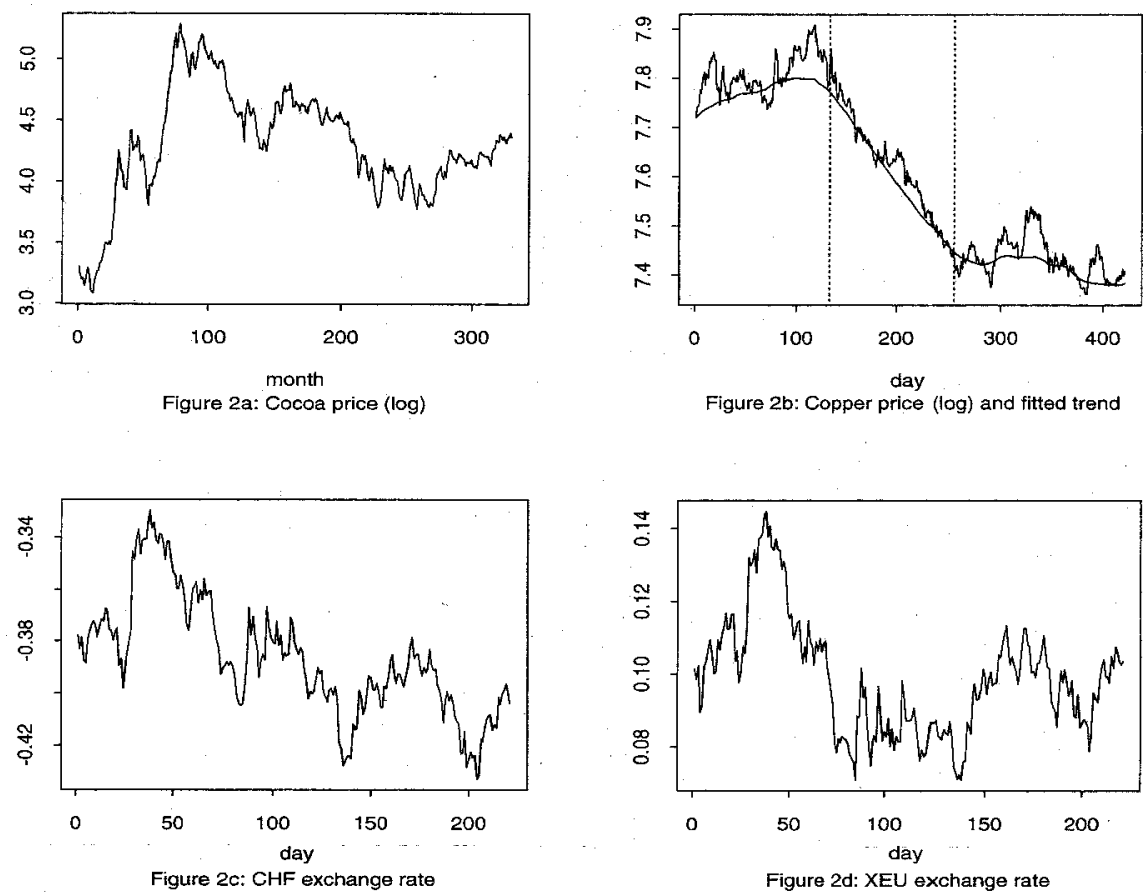

Fig. 2. Monthly prices för cocoa beans (Jan. 1971 - Sept. 1996, $n=310$ ), daily prices for copper (Jan. 2, 1997 - Sept. 2, 1998, $n=421$ ), daily nominal exchange rates for the Swiss Franc $(\log (\mathrm{USD} / \mathrm{CHF}))$ and the European Currency Unit $(\log (\mathrm{USD} / \mathrm{XEU}))$ (Sept. 17, 1997 - Aug. 4, 1998, $n=221$ ). 
First, we fit SEMIFAR models to the observed series. Note in particular that, instead of continuously compounded returns (first difference in natural logarithm of the closing price for consecutive trading days/months), the original series of observed (log-)prices is considered. Thus, in contrast to the traditional approach, it is not assumed a priori that the first integer difference has to be taken to make the series stationary. Instead, the possibilities of stationarity, difference stationarity, deterministic trend, short memory, long memory and antipersistence are left open. It is then decided based on the data which combination of these components may be present.

There has been some discussion in the recent literature about possible unit root behaviour or long memory in financial time series. In view of this, it is interesting to see which hypothesis may be supported by fitting SEMIFAR models. Table 1 summarizes the essential features of the fitted models. The corresponding $95 \%$-confidence intervals are given in brackets. The models were selected using the BIC.

Table 1. Estimation results

\begin{tabular}{l|cc|cc|c}
\hline series & $\hat{d}$ & $95 \%$-c.i. $d$ & $\hat{\phi}_{1}$ & $95 \%$-c.i. $\phi_{1}$ & significant trend \\
\hline cocoa & $.897[.682,1.112]$ & $.394[.142, .646]$ & no \\
copper & $.780[.705, .855]$ & - & - & yes \\
\hline chf & $.913[.810,1.016]$ & - & & no \\
xeu & $.870[.767, .973]$ & - & - & no \\
\hline
\end{tabular}

The estimated value of $d$ and the confidence intervals suggest that all series are nonstationary $(d>1 / 2)$. In addition, the unit roots hypothesis $(d=1)$ can not be rejected for cocoa and chf. On the other hand, for copper and the European Currency Unit, $d=1$ is not contained in the $95 \%$-confidence interval. Thus, for these data, taking the first (integer) difference would lead to overerdifferencing. Furthermore, there is substantial short-term dependence in the cocoa series in form of a strong $\mathrm{AR}(1)$ term.

Since in all cases the estimated value of $m$ was one, testing the presence/ absence of a deterministic trend can be done by testing $H_{o}: g \equiv 0$ against $H_{a}: g \neq 0$. (Note that for $m=1, g$ is the trend function for the first difference.) The only series where $H_{o}$ was rejected (at the $5 \%$ level) was copper. As one may expect (at least a posteriori), for this series, a significant trend is detected due to the relatively long descent in the middle part of the observed time period. The starting and end point of the time interval where $\hat{g}$ exceeded the critical bound are marked in Fig. $2 \mathrm{~b}$ by two vertical lines. Note in particular that fitting a global linear trend would not be appropriate here. For the other three series, apparent local trends do not persist long enough, and can therefore be 'explained' as purely stochastic.

The satisfactory fits of the models are demonstrated by the q-q-plots and correlograms of the residuals in Figs. 3 and 4. Slight departure from normality (for the residuals) can be noticed for the exchange rate data. (Note, however,
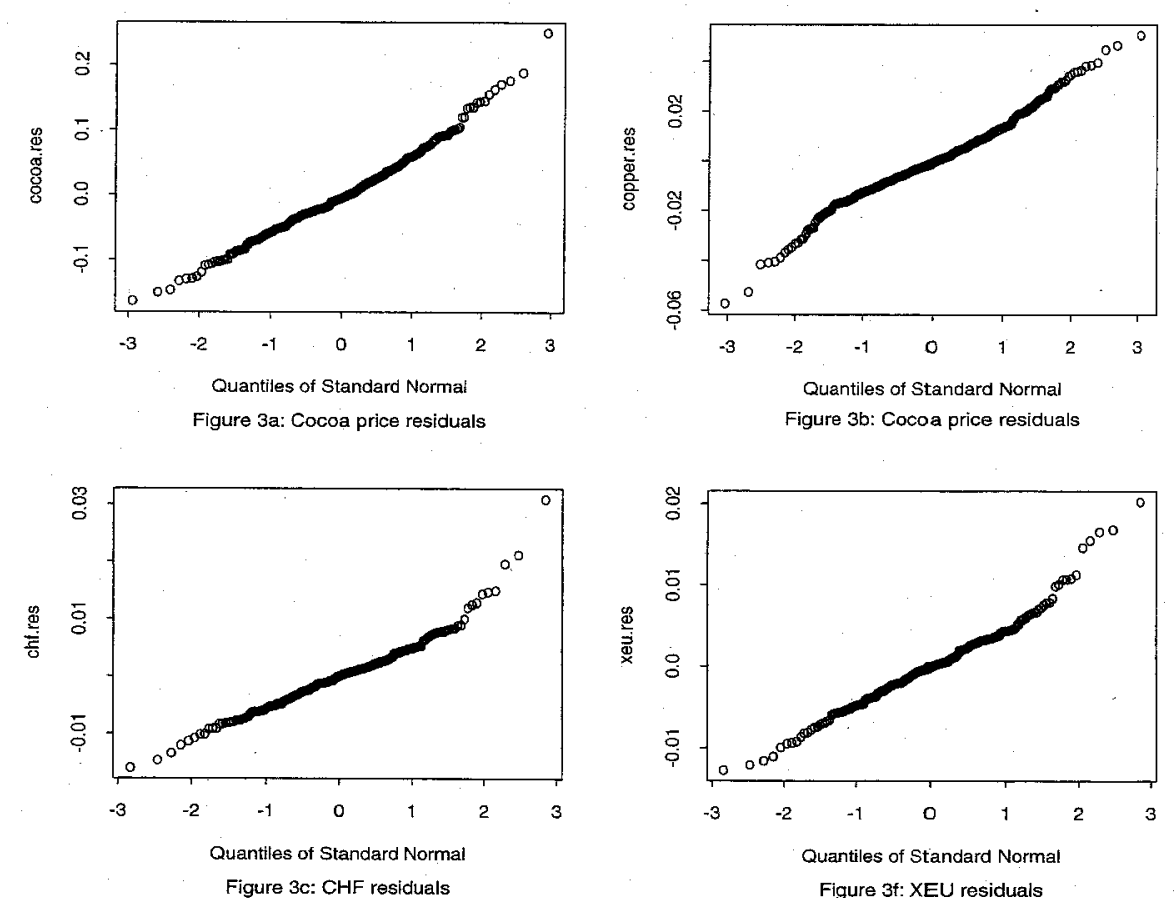

Fig. 3. Normal probability plots of SEMIFAR-residuals for the examples in Figs. 2 a through d.

that normality of $\epsilon_{t}$ is not required for the theoretical results described above to hold.) Also, there is no strong evidence for ARCH (autoregressive conditional heteroskedasticity) errors in the correlograms of the squared residuals (Figs. 4e through $h$ ).

Second, we explore the reliability of forecasts. The $k$-steps ahead out-ofsample forecasts and 95\%- and 99\%-forecast intervals for $k=1,2, \ldots, 20$, using constant extrapolation of $g$, are displayed in Fig. 5. Overall, every future value was inside the $95 \%$ prediction interval. Observe also the weak US dollar in the exchange rate data during the last quarter of the period under consideration. Despite this sudden development, the future values were within the $95 \%$ prediction intervals. It should also be noted that, for $1 / 2<\hat{d}<1$, the width of forecast intervals diverges to infinity at a slower rate than under the unit root hypothesis $d=1$. Thus, shorter forecast intervals are obtained than with unit-root models, such as a random walk. For a detailed discussion see [9]. Clearly, as always with forecasting, sudden extreme structural changes in the behaviour of the data that have not occurred in the past cannot be foreseen (except perhaps with the help of additional information). 
Series : copper.res
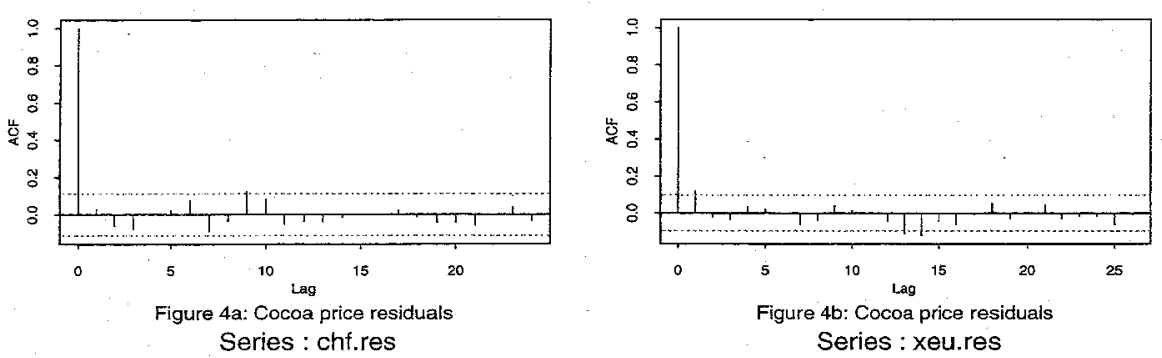

Series : $\operatorname{cocos}^{\wedge} \operatorname{res}^{\wedge}$

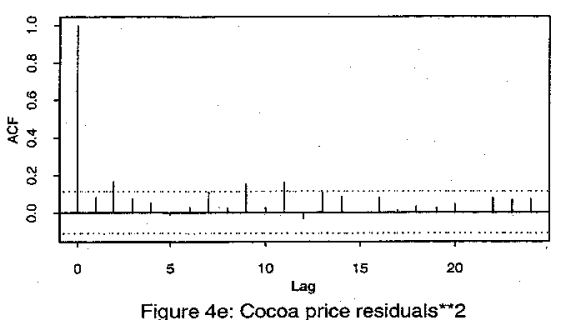

Series : chf.res^2

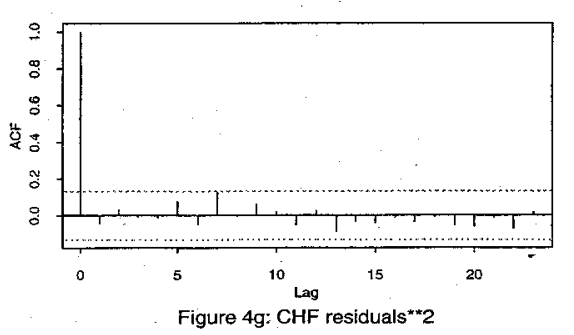

Fig. 4. Autocorrelations of SEMIFAR-residuals (figures $4 \mathrm{a}$ through d) and of the

squared residuals (figures $4 \mathrm{e}$ through $\mathrm{h}$ ) for the examples in figures $2 \mathrm{a}$ through $\mathrm{d}$.
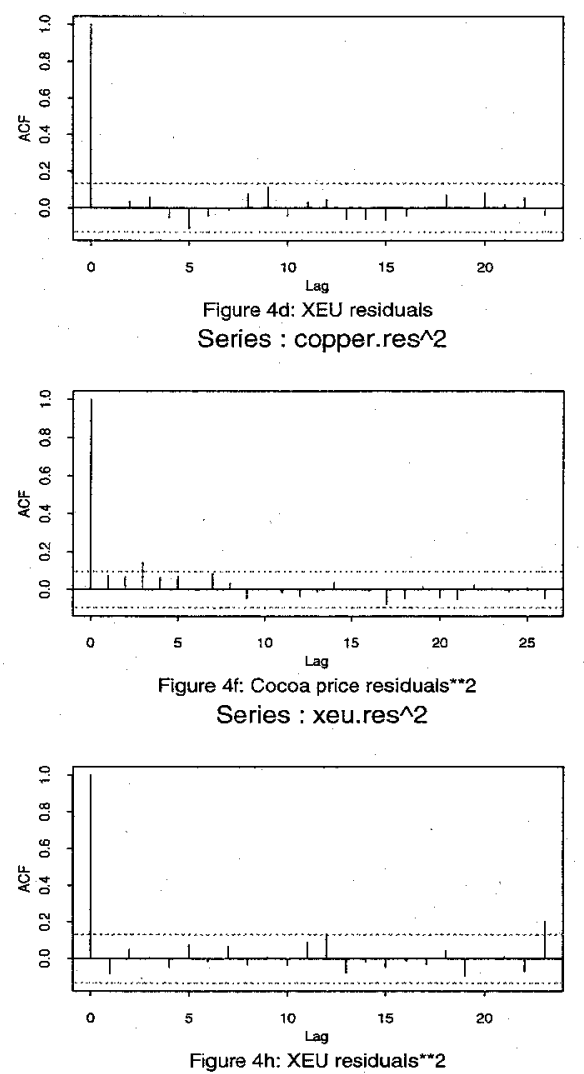
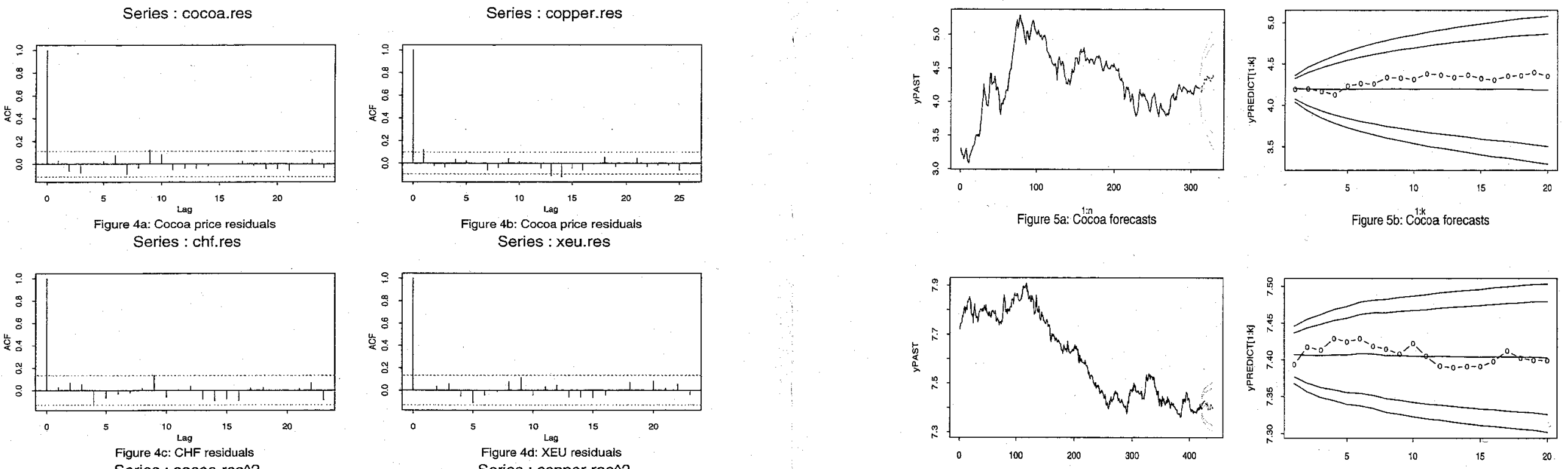

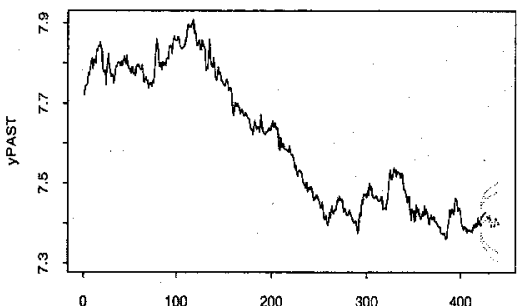

Figure 5c: Copper forecasts

Figure 5d: Copper forecasts

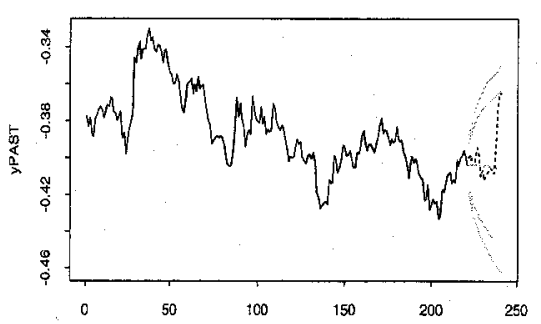

Figure 5e: $\mathrm{CH} F$ forecasts
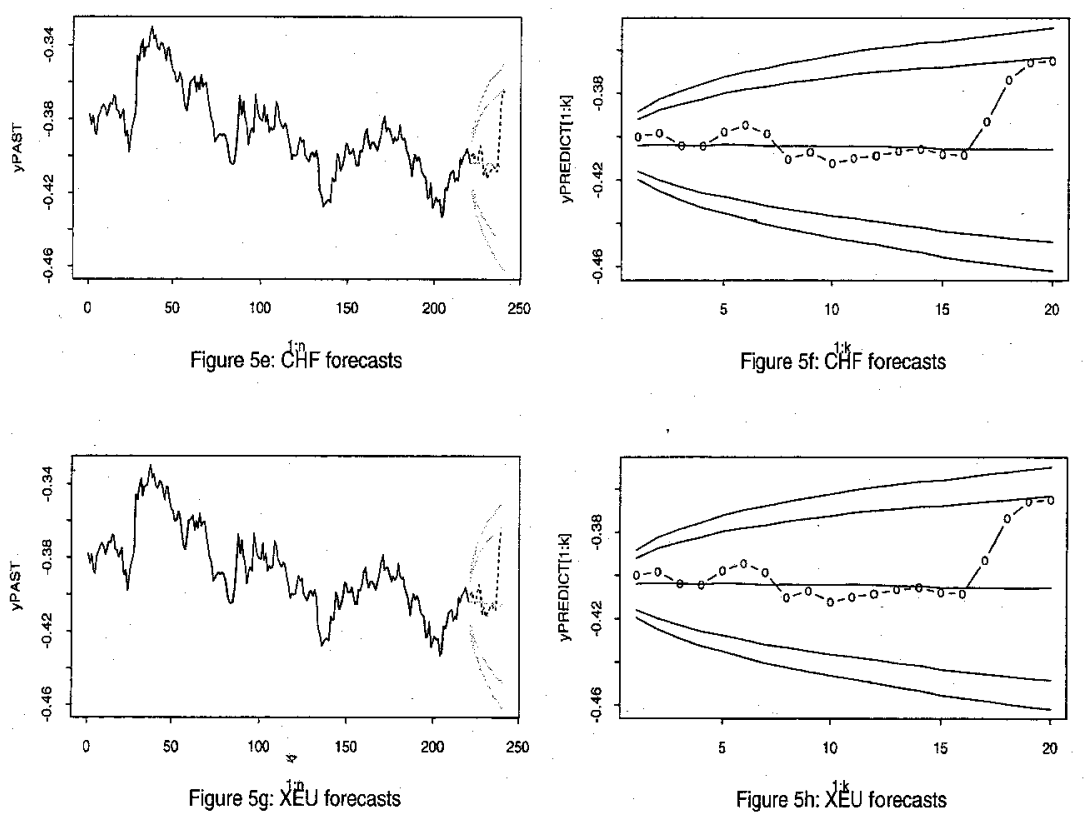

Fig 5. Observed volues with $k$-step ahead SEMIFAR forecasts and $95 \%$ - and $99 \%$ forecast intervals for the examples in figures $2 a$ through $d$. Figures $5 b, d, f$ and $h$ display close-ups of the forecasts and forecast intervals in figures $1 \mathrm{a}, \mathrm{c}$, e and $\mathrm{g}$

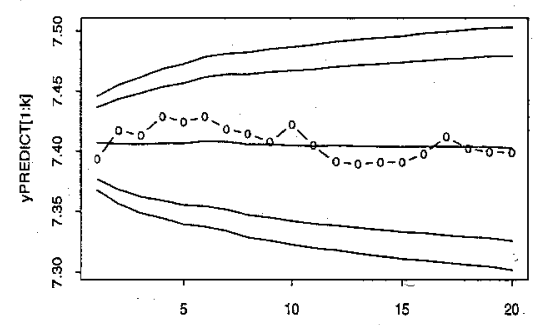




\subsection{Volatility of Stock Market Indices}

Figure $6 \mathrm{a}$ shows daily values of the DAX and the FTSE300 between January 2, 1992 and November 10, 1995 (weekdays only, excluding holidays). The first differences are given in Fig. 6b. Let $I_{t}$ be the original index. To study volatility, we analyze the transformed absolute differences $Y_{t}=\left|I_{t}-I_{t-1}\right|^{\frac{1}{4}}$. The reason for taking the fourth root of the increments is that the marginal distribution of the resulting series is very close to normal (see the normal probability plots in Figs. 6c and d). A similar transformation approach is used, for instance, by [18], [24] and [24]. Ding and Granger found long range dependence in several volatility series that were defined in a similar way. The correlograms of $Y_{t}$ in Figs. $6 \mathrm{e}$ and $\mathrm{f}$ do indeed indicate slowly decaying autocorrelations.

Applying the SEMIFAR method yields $\hat{p}=0$ for both series, $\hat{d}=-0.02$ $[-0.07,0.03]$ for the DAX and $\hat{d}=0.05[0.003,0.100]$ for the FTSE300. In both cases, a significant deterministic trend is found. Fig. 7 shows the two $Y_{t}$ series with the fitted trends and upper and lower $5 \%$ critical limits for testing significance of the trends. The result indicates that there are relatively long periods where volatility is high/low systematically for both series. This extends and is comparable to results by Ding and Granger in the following sense. For stationary long-memory processes, long-term behaviour is determined by the fractional parameter $d$. SEMIFAR models include, apart from $d$, a deterministic (and essentially arbitrary) trend function as an additional building block that can 'explain' long-term fluctuations. A smooth deterministic function can be interpreted as an even stronger (and more systematic) degree of temporal dependence than stationarity with slowly decaying correlations. The significant trends fitted to the volatility series of DAX and FTSE300 thus indicate that there may be even stronger 'long memory' in volatility than suggested by a stationary model with long-range dependence.

A more sophisticated analysis of volatility may be obtained by applying GARCH-type extensions of SEMIFAR models to the original series $I_{t}$. The mathematical theory necessary for such extensions is the subject of current research. For fractional GARCH models that do not include deterministic trend functions see e.g. [3], [17], [24], [32]. In particular, [32] extend the maximum likelihood method of [5] to fractional GARCH models.

\subsection{Simulated Examples}

In this subsection SEMIFAR models are fitted to some simulated series. The series $(n=400)$ are shown in Figs. 1a through d, which are:

Figure 1a: $Y_{i}=X_{i}$ where $X_{i}$ is a fractional autoregressive process of oder $p_{0}=0$ with $d^{0}=0.4$

Figure 1b: $Y_{i}=g(t)+X_{i}$ where $X_{i}$ is a fractional autoregressive process of oder $p_{0}=0$ with $d^{0}=0.4$ (but not the same realization as in figure 1a) and $g(t)=1.75 *\left(1 /\left(1+e^{4-8 t}\right)-\sin (2 \pi t)\right)$.

Figure $1 \mathrm{c}: Y_{i}-Y_{i-1}=X_{i}$ where $X_{i}$ is a fractional autoregressive process of oder $p_{0}=0$ with $d^{0}=-0.3$
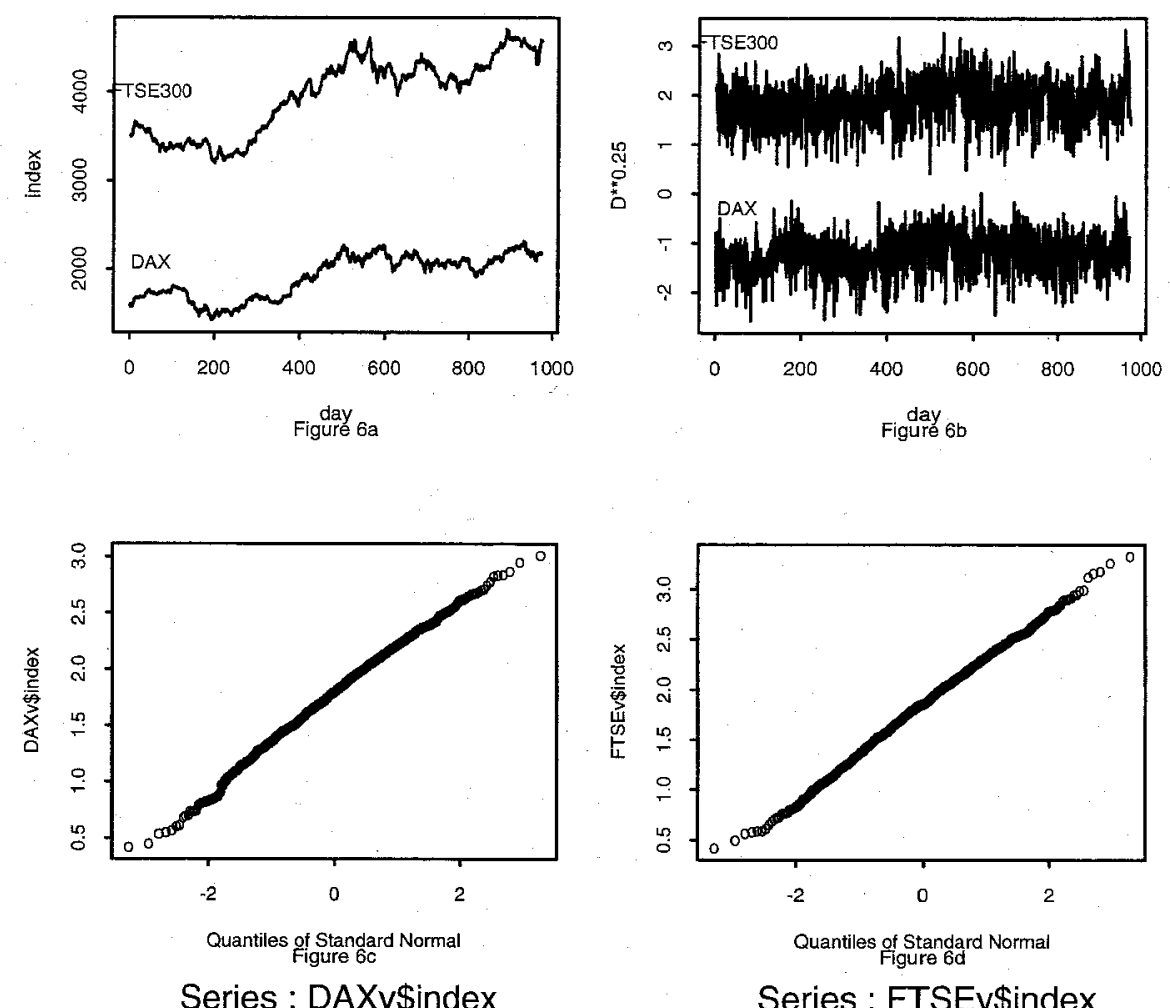

Series: FTSEv\$index
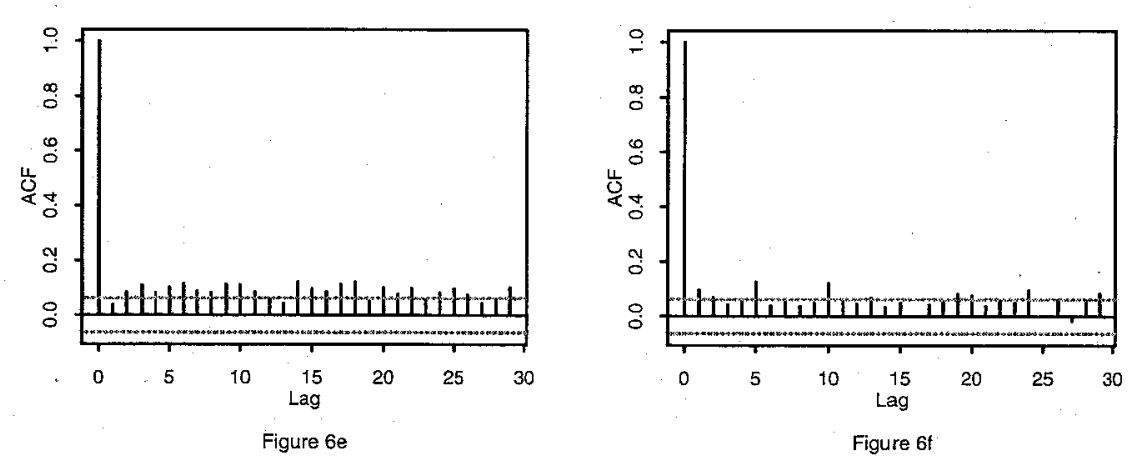

Fig. 6. Daily DAX and FTSE300 values $I_{t}$ (Fig, 6a), first difference (Fig. 6b), normal probability plots of $Y_{t}=\left|I_{t}-I_{t-1}\right|^{\frac{1}{4}}$ (Figs. 6c,d) and autocorrelations of $Y_{t}$ (Figs. 6e,f) 


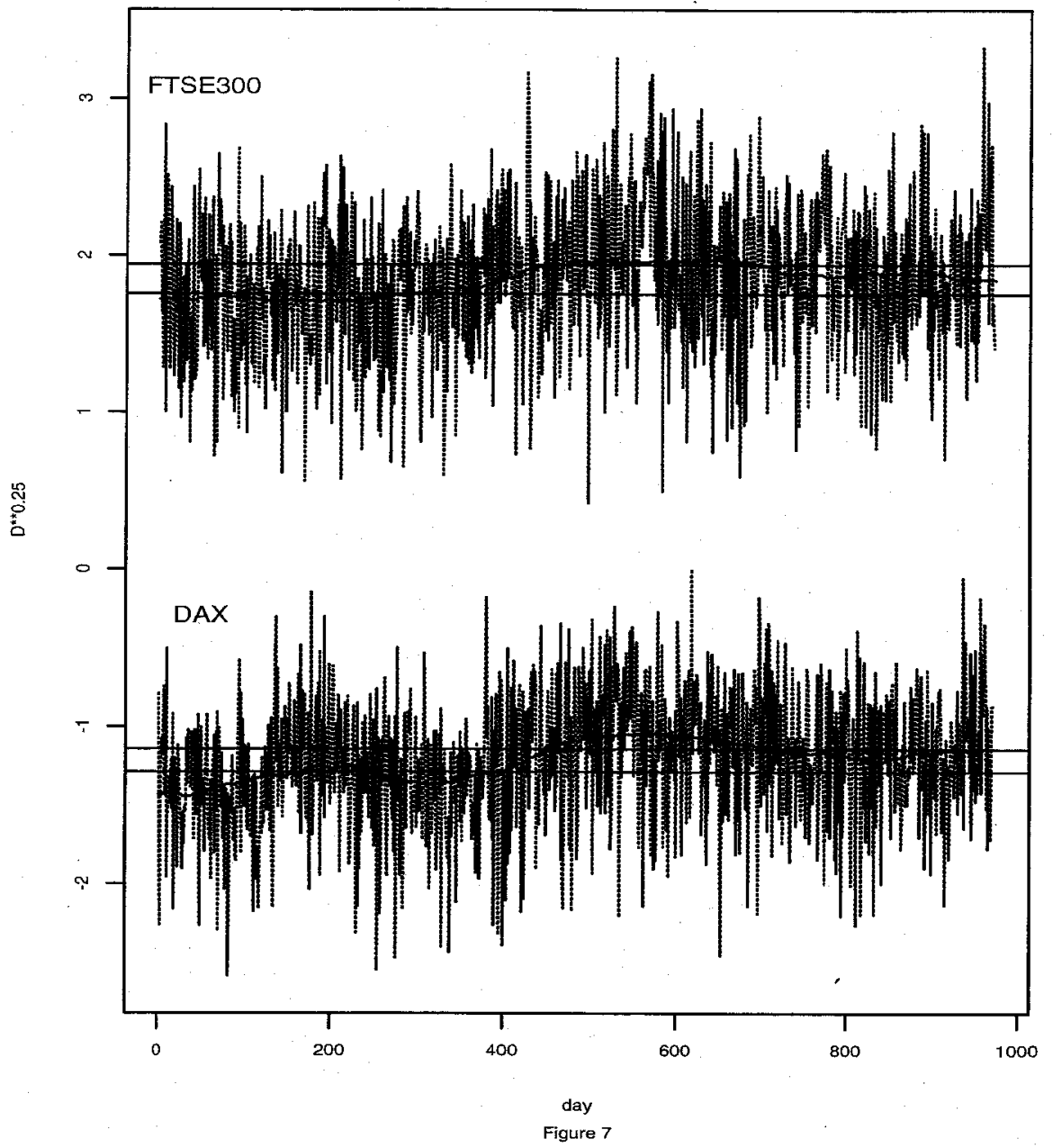

Fig. 7. Trends fitted by the SEMIFAR method to $Y_{t}=\left|I_{t}-I_{t-1}\right|^{\frac{1}{4}}$ where $I_{t}=D A X$ and FTSE300 respectively. Also given are the $5 \%$ rejection limits for testing where the trend is significant.

Figure 1d: $Y_{i}-Y_{i-1}=g(t)+X_{i}$ where $X_{i}$ is the same fractional autoregressive process as in Fig. 1c and $g(t)=0.2 *(t-0.5)$.

All of these simulated series were generated by S-Plus with the "error" series $X_{i}$ generated by the function arima.fracdiff.sim. Since a visual assessment of the time series plots appears to be difficult, it is interesting to see in how far the proposed method provides better information. The estimates $\hat{p}$ and $\hat{\eta}=\hat{d}$ (because of $p_{0}=0$ ) together with $95 \%$-confidence intervals, obtained by fitting SEMIFAR models for $p=0,1,2,3,4,5$ and choosing $p$ based on the BIC, are
Table 2. Estimates of $p_{o}, d^{\circ}$ and $m^{\circ}=\left[d^{\circ}+0.5\right]$ for the four simulated examples in Figs. 1a through 1d. The true values of $p_{o}, d^{\circ}$ and $m^{\circ}$ are given in brackets. Also given are the $95 \%$-confidence intervals for $d^{o}$ and the results of the testing on whether there is a significant trend $g$ in the data.

\begin{tabular}{l|c|c|cc|c}
\hline Figure & $\hat{p}\left(p_{o}\right)$ & $\hat{m}\left(m^{\circ}\right)$ & $\hat{d}\left(d^{\circ}\right)$ & $95 \%$-C.I. for $d^{\circ}$ & testing on $g$ \\
\hline Fig. 1a & $0(0)$ & $0(0)$ & $0.425(0.4)$ & {$[0.348,0.502]$} & not significant \\
Fig. 1b & $0(0)$ & $0(0)$ & $0.329(0.4)$ & {$[0.252,0.406]$} & significant \\
Fig. 1c & $0(0)$ & $1(1)$ & $0.764(0.7)$ & {$[0.687,0.841]$} & not significant \\
Fig. 1d & $0(0)$ & $1(1)$ & $0.762(0.7)$ & {$[0.685,0.839]$} & significant \\
\hline
\end{tabular}

given in Table 2. Also given are the 95\%-confidence intervals for $d^{\circ}=\left[d^{\circ}+0.5\right]$ and the results of the testing whether there is a significant trend $g$ in the data.

The values of $\hat{m}$ and $\hat{p}$ are correct for all four series. Thus, in particular, the method yields the correct answer to the question whether differencing is needed i.e. whether the observed series has a stochastic trend component. Moreover, the estimates $\hat{\eta}$ are very close to the true values and the true values are always in the confidence intervals. Similarly, regarding the presence of a deterministic trend component, the results give correct indications. Hence the proposed models provide a way to distinguish stochastic trends, deterministic trends, long- and short memory or mixtures of these. It can be expected that more refined smoothing methods, such as local bandwidth choice (see e.g. [11]), may lead to even better estimates of $g$. This will be pursued elsewhere.

\subsection{Comparison Between SEMIFAR and AR}

In this section a brief comparison between the SEMIFAR model and the well known AR model will be made using the four examples in Sect. 5.1. Using the S-PLUS function arima.mle and the AIC criterion, an AR(2) model $y_{t}=$ $0.3392 y_{t-1}-0.0896 y_{t-2}+\epsilon_{t}$ with $\hat{\sigma}^{2}=0.00402$ was obtained for the cocoa data The AR model obtained for the xeu data was $y_{i}=-0.1178 y_{t-1}+\epsilon_{t}$ of order 1 with $\hat{\sigma}^{2}=0.000027$. The other two data sets copper and chf were shown to be white noises. The ratios between the widths of prediction intervals for the $k$-step forecasting obtained be the fitted SEMIFAR model and the AR one are given in Table 3.

Note in particular that, in 'stationary versus unit root' approaches, a decision has to be made between $d=0$ and $d=1$. A wrong decision has an extreme impact on forecast intervals, since the width of forecast intervals is asymptotically

Table 3. Ratios of prediction intervals by SEMIFAR model and AR one

\begin{tabular}{l|cccccccccccc}
\hline$k$ & 1 & 2 & 3 & 4 & 5 & 6 & 7 & 8 & 9 & 10 & 15 & 20 \\
\hline cocoa & 1.00 & 0.97 & 0.98 & 0.99 & 0.99 & 0.99 & 0.98 & 0.98 & 0.97 & 0.97 & 0.94 & 0.92 \\
copper & 0.98 & 0.88 & 0.82 & 0.77 & 0.74 & 0.71 & 0.69 & 0.67 & 0.66 & 0.64 & 0.59 & 0.56 \\
chf & 1.00 & 0.96 & 0.93 & 0.91 & 0.89 & 0.88 & 0.87 & 0.86 & 0.86 & 0.85 & 0.82 & 0.80 \\
xeu & 1.00 & 0.99 & 0.96 & 0.94 & 0.92 & 0.91 & 0.89 & 0.88 & 0.87 & 0.86 & 0.82 & 0.79 \\
\hline
\end{tabular}


constant for $d=0$ whereas it diverges to infinity at the rate $\sqrt{k}$ for $d=1$. In contrast, for FARIMA models, prediction intervals are of order $O\left(k^{\tau / 2}\right)$ with $\tau$ varying in a continuous range, including $\tau=0$ and $\tau=1$ as special cases. The value of $\tau=\max \{0,2 d-1\}$ is estimated from the data by maximum likelihood and the extreme decision between $O(1)$ and $O\left(k^{.5}\right)$ is avoided. As a result, prediction intervals are better adapted to the observed data, and often shorter if there is antipersistent. This is, in particular, often the case for foreign exchange rates. Consider for example the results in [9] on forecasting nominal exchange rates. There, the most dramatic improvement was achieved for the British Pound. Already for $k=20$, the average interval was shorter by a factor of about 0.7 , while the coverage probability of the interval appeared to be correct. Similar results were obtained in a recent $\mathrm{PhD}$. thesis of [36], who found, in comparison, shorter prediction intervals for eight (out of eight) nominal foreign exchange rates. Many of them were shorter by a factor clearly smaller than 0.9 for $k=20$ (see [36]). Further evidence of antipersistence in financial time series can also be found in [36]. In contrast to foreign exchange rates (and commodities), [36] found that traditional [10] ARIMA forecast intervals are typically too optimistic (i.e. too short) if the degree of persistence is strong, such as for nominal stock market indices.

\section{Final Remarks}

In this paper, we summarized recent results on so-called SEMIFAR models for time series that incorporate stochastic trends, deterministic trends, long-range dependence and short-range dependence. The potential usefulness of this model for economic time series analysis is illustrated by several data examples. In particular, the proposed method helps the data analyst to answer the question which of these components are present in the observed series. How well the different components can be distinguished depends on the specific process and, in particular, on the shape of the trend function. Therefore, in order that the proposed method is effective in general, the observed series must not be too short. In cases where one has sufficient a priori knowledge about the type of trend (e.g. linear, exponential etc.), parametric trend estimation is likely to provide more accurate results. This can be done simply by replacing the general function $g$ in Definition (1) by the corresponding parametric function.

Further refinements of the method, such as local polynomial fitting of $g$, local bandwidth choice (see e.g. [11]), bootstrap confidence intervals, faster algorithms (see [22]) or other smoothing methods, etc., will be worth pursuing in future. Also, various extensions of SEMIFAR models are possible. For instance, as for classical ARIMA models, stochastic seasonal components can be included by multiplying the left hand side of (1) by a polynomial $\phi_{\text {seas }}(B)=\sum \phi_{j, s e a s} B^{s j}$ where $s \in N$ is the seasonal period. Other extensions, such as inclusion of parametric and nonparametric explanatory variables, other seasonal components and nonlinearities in the stochastic part of the process, are the subject of current research.

\section{Acknowledgements}

This research was supported in part by an NSF grant to MathSoft, Inc. (Seattle), and by the Center of Finance and Econometrics, University of Konstanz, Germany. The data for the exchange rates were obtained from the Web-site of PACIFIC (Policy Analysis Computing \& Information Facility in Commerce) at the University of British Columbia, Vancouver, Canada; the prices of copper is from the homepage of the London Metal Exchange, and the cocoa price series from the ICCO's (International Cocoa Organization) Web-page. We would like to thank the authors of these data sets for making their data publicly available. Finally, we would like to thank Dr. Elke M. Hennig (Citibank, Frankfurt) for the stock market series.

\section{References}

1. H. Akaike: Biometrika 66, 237 (1979)

2. N. S. Altman: Journal of the American Statistical Association 85, 749 (1990)

3. R. T. Bailie, T. Bollerslev and H. O. Mikkelsen: Journal of Econometrics 74, 3 (1996)

4. J. Beran: Statistics for long-memory processes (Chapman and Hall, New York 1994)

5. J. Beran: Journal of the Royal Statistical Society, series B 57, 695 (1995)

6. J. Beran: SEMIFAR models - a semiparametric fractional framework for modelling trends, long-range dependence and nonstationarity (Preprint, University of Konstanz 1999)

7. J. Beran, R. J. Bhansali and D. Ocker: On unified model selection for stationary and nonstationary short and long-memory autoregressive processes (To appear in Biometrika 1998)

8. J. Beran and Y. Feng: Local polynomial fitting with long-memory, short-memory and antipersistent errors, The Ann. Instit. Statist. Math. (in press) (2002)

9. J. Beran and D. Ocker: J. Statist. Plann. Infer. 80, 137 (1999)

10. G. E. Box and G. M. Jenkins: Time Series Analysis: Forecasting and Control (Holden Day, San Francisco 1976)

11. M. Brockmann: Journal of the American Statistical Association 88, 1302 (1993)

12. Y. W. Cheung: Journal of Business and Economic Statistics 11, 93 (1993)

13. S. T. Chiu: Statistics and Probability Letters 8, 347 (1989)

14. D. R. Cox: 'Long-range dependence: a review'. In: Statistics: An Appraisal, Proceedings 50th Anniversary Conference, ed. by H. A. David and H. T. David (The Iowa State University Press 1984) 55

15. Csörgö, S. J. Mielniczuk: Annals of Statistics 23, 1000 (1995)

16. P. J. Diggle: Time Series - a biostatistical introduction (Oxford University Press, Oxford 1990)

17. Z. Ding and C. W. J. Granger: Journal of Econometrics 73, 185 (1996)

18. Z. Ding, C. W. J. Granger and R. F. Engle: Journal of Empirical Finance 1, 83 (1993)

19. W. M. Fong and S. Ouliaris: Journal of Applied Econometrics 10, 255 (1995)

20. G. Franke, R. Stapleton and M. G. Subrahmanyan: When are options overpriced? The Black-Shcholes model and alternative characterisations of the pricing kernel (Forthcoming in European Finance Review, 1999) 
21. T. Gasser and H. G. Muller: 'Kernel estimation of regression functions'. In: Smoothing Techniques for Curve Estimation, ed. by T. Gasser and M. Rosenblatt, Lecture Notes in Mathematics, (Springer, New York 1979) 757, pp.23-68

22. T. Gasser, A. Kneip, W. Köhler: Journal of the American Statistical Association 86, 643 (1991)

23. C. W. J. Granger: Journal of Econometrics 14, 227 (1980)

24. C. W. J. Granger and Z. Ding: Journal of Econometrics 73, 61 (1996)

25. C. W. J. Granger and R. Joyeux: Journal of Time Series Analysis 1, 15 (1980)

26. P. Hall and J. Hart: Stochastic Processes and Their Applications 36, 339 (1990)

27. F. R. Hampel: 'Data analysis and self-similar processes'. Proceedings of the 46th Session of ISI ( Tokyo, Book 4 1987) pp. 235-254

28. E. J. Hannan and B. G. Quinn: Journal of the Royal Statistical Society, series B 41, 190 (1979)

29. E. Herrmann, T. Gasser, and A. Kneip: Biometrika 79, 783 (1992)

30. J. R. M. Hosking: Biometrika 68, 165 (1981)

31. H. Künsch: Proc. First World Congress of the Bernoulli Society, Tashkent Vol 1, $67(1986)$

32. S. Q. Ling and W. K. Li: JASA 92, 1184 (1997)

33. C. Y. Liu, and J. He: Journal of Finance 36, 773 (1991)

34. B. B. Mandelbrot: The Review of Economics and Statistics 53, 225 (1971)

35. B. B. Mandelbrot: The fractal geometry of nature (Freeman, New York 1983)

36. D. Ocker: Stationary and nonstationary fractional ARIMA models - model choice, forecasting, aggregation and intervention. Unpublished $\mathrm{PhD}$ thesis, University of Konstanz (1999)

37. M. B. Priestley: Spectral Analysis and Time Series (Academic Press, London 1981)

38. B. K. Ray and R. S. Tsay: Biometrika 84, 791 (1997)

39. L. C. G. Rogers: Mathematical Finance 7, 95 (1997)

40. P. A. Samuelson: Industrial Management Review VI, 41 (1965)

41. G. Schwarz: Annals of Statistics 6, 461 (1978) 